\title{
Das Recht am eigenen Bild - Vorsicht bei Patientenfotos und Videodokumentation im Vortrag, bei Publikationen oder auf der Praxishomepage
}

\author{
Albrecht Wienke ${ }^{1}$ \\ Rosemarie Sailer ${ }^{1}$
}

1 Wienke \& Becker - Köln

\section{Text}

Bei medizinisch-wissenschaftlichen Vorträgen, bei ärztlichen Fort- und Weiterbildungsveranstaltungen, in Fachzeitschriften und auch in der medizinischen Forschung werden regelmäßig Abbildungen von Patienten zur Veranschaulichung von Krankheitsbildern, Behandlungsverfahren oder intraoperativer Situationen verwendet. Dies geschieht in Form der Präsentation von Röntgenbildern, intraoperativen Foto- oder Videoaufnahmen oder Bildern von Körperteilen. Auch bei der Darstellung einzelner ärztlicher Tätigkeiten oder Behandlungsverfahren ist es jedenfalls für bestimmte Eingriffe zulässig, Vorher- und Nachher-Bilder von Patienten auf der Praxishomepage zu veröffentlichen oder in der Praxis oder bei Vorträgen vorzustellen. Die Verwendung und Präsentation solcher Bilder von Patienten oder Abbildungen von Körperteilen ist gerade in der ärztlichen Ausbildung, bei Vorträgen und auch in wissenschaftlichen Publikationen geradezu an der Tagesordnung und selbstverständlich. Dennoch sollte man sich dabei im Klaren darüber sein, dass die Rechte der abgebildeten Persönlichkeiten und derjenigen, welche die Abbildungen (Fotos, Röntgenbilder etc.) hergestellt haben, nicht verletzt werden dürfen. Welche Aspekte hierbei zu beachten sind und wie man sich vor Ansprüchen Dritter auf Unterlassung und Schadensersatz am besten schützen kann, stellen wir im nachfolgenden Beitrag vor:

\section{Urheberrecht des Arztes}

Der Arzt, der Aufnahmen vom Körper oder Körperteilen eines Patienten macht, gilt rechtlich betrachtet als Urheber sogenannter Lichtbilder und erwirbt damit umfassende Nutzungs- und Verfügungsrechte. Diese umfassen insbesondere auch die Nutzung, d.h. vor allem die Vervielfältigung sowie Veröffentlichung dieser Aufnahmen. Geregelt ist dies im Einzelnen im Urheberrechtsgesetz (UrhG). Das bedeutet aber nicht, dass solche selbst hergestellten Bilder beliebig verwendet werden dürften: Eingeschränkt werden die Rechte des Urhebers durch das sog. Recht am eigenen Bild der abgebildeten Person. Dies bedeutet, dass eine Veröffentlichung bzw. Verwendung der Patientenfotos nur dann zulässig ist, wenn die Rechte des jeweiligen Patienten nicht verletzt werden. Das Recht am eigenen Bild geht zurück auf das vom Grundgesetz geschützte allgemeine Persönlichkeitsrecht und findet seine gesetzliche Ausprägung in $\S 22$ des Kunsturhebergesetzes (KUG). Diese Regelung schreibt vor, dass Bildnisse lediglich mit Einwilligung des Abgebildeten verbreitet oder öffentlich zur Schau gestellt werden dürfen.

\section{Einwilligung des Patienten immer erforderlich?}

Dies heißt nichts anderes, dass grundsätzlich die Einwilligung des Patienten vorliegen muss, wenn ein Foto von inm, sei es im Rahmen eines Vortrags vor Fachkreisen, der Werbung auf der Praxishomepage oder in sonstiger Weise veröffentlicht werden soll.

Dies gilt jedoch nicht in jedem Fall: Die Rechtsprechung sieht ein schutzwürdiges Interesse des Abgebildeten immer nur dann, wenn er in einer für Dritte erkennbaren Weise wiedergegeben wird. Ist also der Patient auf dem Foto nicht erkennbar und auch sein Name nicht veröffentlicht, besteht nicht die Verpflichtung, dessen Einwilligung einzuholen. In Fällen, in denen intraoperativ Bilder von Gewebe, Knochen oder Organen gemacht werden, ist daher keine Einwilligung des Patienten erforderlich, wenn diese Bilder anonym veröffentlicht werden.

Vorsicht ist jedoch geboten, wenn Fotos vom äußeren Erscheinungsbild des Patienten gemacht werden (z.B. bei Erkrankungen oder Behandlungsverfahren, bei denen die Gesichtszüge des Patienten erkennbar sind). Selbst wenn Teile des Gesichts (z.B. durch Balken oder Verpixeln) unkenntlich gemacht werden, sollte bei solchen Bildern stets die schriftliche Einwilligung des Patienten eingeholt werden. Denn hier ist die Rechtsprechung streng: Nach einer Entscheidung des Oberlandesgerichts Düsseldorf vom 09.02.2010 (I 20 U 151/09) genügt es, dass die Erkennbarkeit für einen mehr oder weniger großen Bekanntenkreis besteht. Zudem kann sich die Erkennbarkeit nach einem Urteil des OLG Zweibrücken vom 07.06.2010 (4 W 53/10) auch aus sonstigen Merkmalen des Bildes, dem beigefügten Text oder dem 
Zusammenhang mit früheren Veröffentlichungen ergeben. Bei Fotos, auf denen Personen vollständig bzw. das Gesicht abgebildet ist, ist daher die Einwilligung des Patienten erforderlich. Gleiches gilt für Fälle, in denen aufgrund besonderer körperlicher Merkmale, wie etwa auffälliger Narben, Amputationen, Pigmentierungen, Tätowierungen etc. jedenfalls Rückschlüsse auf die Person des Abgebildeten möglich sind. Auch bei Röntgenbildern kann es in Einzelfällen möglich sein, aufgrund spezifischer anatomischer Besonderheiten Rückschlüsse auf die Person des Patienten zu ziehen, in aller Regel wird eine Erkennbarkeit aber nicht zu befürchten sein.

\section{Datenschutzrechtliche Legitimation?}

$\S 28$ Abs. 8 i. V. m. Abs. 6 Nr. 4 Bundesdatenschutzgesetz (BDSG) erlaubt eine Erhebung, Verarbeitung und Nutzung von Gesundheitsdaten, zu denen Röntgenbilder und $\mathrm{Pa}$ tientenfotos zählen, zwar auch ohne Einwilligung des Patienten, wenn dies zur Durchführung wissenschaftlicher Forschung erforderlich ist und das wissenschaftliche Interesse an der Durchführung des Forschungsvorhabens das Interesse des Betroffenen an dem Ausschluss der Erhebung, Verarbeitung und Nutzung überwiegt. Hieraus kann sich also in Einzelfällen die Berechtigung ergeben, auch ohne eine ausdrückliche Einwilligung des Patienten Bilder für die wissenschaftliche Forschung zu verwenden, was allerdings im Einzelnen vom Arzt zu beweisen wäre. Für die bloße Veranschaulichung oder Werbung gilt dies in jedem Fall nicht.

\section{Rechtliche Konsequenzen einer unzulässigen Veröf- fentlichung}

In Zweifelsfällen gilt: Besser die Einwilligung des Patienten einholen, da bei einer nicht autorisierten Veröffentlichung trotz Erkennbarkeit des Patienten auch eine Verletzung der ärztlichen Schweigepflicht und damit eine Strafbarkeit, des weiteren auch berufsrechtliche Konsequenzen für den Arzt im Raum stehen. Dem Patienten können Schadensersatz- und Schmerzensgeldansprüche zustehen, des Weiteren kann er die Löschung der veröffentlichten Bilder auf Kosten des Veranlassers verlangen. Da der Arzt die Beweislast dafür trägt, dass er zur Veröffentlichung der Fotos berechtigt ist, sollte er daher auch in Zweifelsfällen - also immer dann, wenn eine Erkennbarkeit des Patienten nicht ganz auszuschließen ist - dessen Einwilligung einholen. Dies sollte aus Nachweisgründen am besten schriftlich erfolgen. Von einer Einwilligungsklausel in Allgemeinen Geschäfts- oder Krankenhausaufnahmebedingungen ist dagegen abzuraten. Zwar gibt es hierzu noch keine einheitliche Rechtsprechung, jedoch dürfte eine solche Klausel für die meisten Patienten überraschend und damit unwirksam sein. Im Übrigen muss die schriftliche Einwilligung des Patienten keine besondere Form haben; sie muss allerdings Hinweise darüber enthalten, auf welche Art von Bildern oder Abbildungen sich die Einwilligung zur Veröffentlichung bezieht, in welchen Medien die Publikation erfolgen soll und dass ein jederzeitiger Widerruf der erteilten Einwilligung mög lich ist.

\section{Vorsicht vor unbefugter Nutzung fremder Bilder}

Ungeachtet der Frage, ob möglicherweise mit der Veröffentlichung das Recht am eigenen Bild des Patienten verletzt wird, ist im Vorfeld immer zu prüfen, wem das Urheberrecht und etwaige Nutzungsrechte an den Bildern zustehen. Denn in den seltensten Fällen wird der Arzt das Röntgenbild tatsächlich selbst angefertigt haben und auch bei intraoperativen Aufnahmen führt häufig ein Assistent den Kameratrokar. Urheber im Rechtssinn ist aber immer derjenige, der auf den Auslöser drückt bzw. die wesentlichen Parameter - Lage, Einstellung, Ausschnitt etc. - vorgibt, also bei Röntgenbildern in aller Regel eine MTA-R. Das Urheberrecht ist nicht übertragbar, jedoch werden die Nutzungs- und Verwertungsrechte an den entstandenen Bildern stillschweigend auf den Arbeitgeber, also den Praxisinhaber oder das Krankenhaus, übertragen. Will daher ein Arzt diese Bilder nutzen oder veröffentlichen, muss er sich diesbezügliche Nutzungsrechte erst einräumen lassen. Eine Ausnahme gilt, wenn die Bilder im Rahmen einer wahlärztlichen Leistung oder Privatambulanz oder in der eigenen Praxis eines niedergelassenen Arztes entstehen, da die Nutzungsrechte dann dem betreffenden Arzt unmittelbar zustehen.

\section{Fazit}

Bei der Veröffentlichung von Patientenfotos oder Röntgenbildern gilt folgende Regel:

Zum einen ist zu prüfen, ob und inwieweit eine Legitimation zur Veröffentlichung erforderlich ist. Dies gilt in Bezug auf Urheber- und Nutzungsrechte, da diese häufig der jeweiligen klinischen Einrichtung zustehen, in der die (Röntgen-) Bilder erstellt worden sind und in der der Arzt angestellt ist.

Zum anderen ist in Bezug auf schutzwürdige Rechte des Patienten zu prüfen, ob und inwieweit durch die Veröffentlichung der Abbildungen Rückschlüsse auf die Person des Patienten möglich sind. Ist dies der Fall oder jedenfalls in allen Zweifelsfällen, ist eine schriftliche Einwilligung des Patienten einzuholen. Häufig wird es sich aber um (intraoperative oder radiologische) Bilder von einzelnen Organen, Körperteilen oder Knochen handeln, so dass - die erforderliche Anonymisierung vorausgesetzt keinerlei Hinweise auf die Identität des Patienten gegeben werden. In diesem Fall können die Bilder ohne Information und ohne Einwilligung des Patienten verwendet und veröffentlich werden.

\section{Korrespondenzadressen:}

Dr. iur. Albrecht Wienke

Wienke \& Becker - Köln, Sachsenring 6, 50677 Köln

Awienke@Kanzlei-WBK.de

LL.M. Medizinrecht Rosemarie Sailer

Wienke \& Becker - Köln, Bonner Straße 323, 50968 Köln

rsailer@kanzlei-wbk.de 
Bitte zitieren als

Wienke A, Sailer R. Das Recht am eigenen Bild - Vorsicht bei Patientenfotos und Videodokumentation im Vortrag, bei Publikationen oder auf der Praxishomepage. GMS Mitt AWMF. 2017;14:Doc1. DOI: 10.3205/awmf000319, URN: urn:nbn:de:0183-awmf0003193

Artikel online frei zugänglich unter

http://www.egms.de/en/journals/awmf/2017-14/awmf000319.shtml
Eingereicht: 05.12.2017

Veröffentlicht: 06.12.2017

\section{Copyright}

(C)2017 Wienke et al. Dieser Artikel ist ein Open-Access-Artikel und steht unter den Lizenzbedingungen der Creative Commons Attribution 4.0 License (Namensnennung). Lizenz-Angaben siehe http://creativecommons.org/licenses/by/4.0/. 